\title{
Separate but interacting recognition memory systems for different senses: The role of the rat perirhinal cortex
}

\author{
Mathieu M. Albasser, ${ }^{1,3}$ Eman Amin, ${ }^{1}$ Mihaela D. Iordanova, ${ }^{1}$ Malcolm W. Brown, ${ }^{2}$ \\ John M. Pearce, ${ }^{1}$ and John P. Aggleton ${ }^{1}$ \\ ${ }^{1}$ School of Psychology, Cardiff University, Cardiff, CF10 3AT, Wales, United Kingdom; ${ }^{2}$ MRC Centre for Synaptic Plasticity, \\ Department of Physiology and Pharmacology, University of Bristol, Medical School, Bristol, BS8 1TD, United Kingdom
}

\begin{abstract}
Two different models (convergent and parallel) potentially describe how recognition memory, the ability to detect the re-occurrence of a stimulus, is organized across different senses. To contrast these two models, rats with or without perirhinal cortex lesions were compared across various conditions that controlled available information from specific sensory modalities. Intact rats not only showed visual, tactile, and olfactory recognition, but also overcame changes in the types of sensory information available between object sampling and subsequent object recognition, e.g., between sampling in the light and recognition in the dark, or vice versa. Perirhinal lesions severely impaired object recognition whenever visual cues were available, but spared olfactory recognition and tactile-based object recognition when tested in the dark. The perirhinal lesions also blocked the ability to recognize an object sampled in the light and then tested for recognition in the dark, or vice versa. The findings reveal parallel recognition systems for different senses reliant on distinct brain areas, e.g., perirhinal cortex for vision, but also show that: (1) recognition memory for multisensory stimuli involves competition between sensory systems and (2) perirhinal cortex lesions produce a bias to rely on vision, despite the presence of intact recognition memory systems serving other senses.
\end{abstract}

Consider the problem of determining whether an object you encounter is the same, or different, from a previously encountered object. This recognition problem is important as novel stimuli require rapid identification so that they can become the focus of attention. The perirhinal cortex is vital for visual recognition memory (Murray and Mishkin 1986; Mumby and Pinel 1994; Brown and Aggleton 2001; Winters et al. 2008), but also receives convergent information from other senses (Burwell and Amaral 1998; Furtak et al. 2007). Consequently, the perirhinal cortex might have a general role (Convergent Model) (Fig. 1A), making it important for the recognition of information from multiple sensory modalities (Otto and Eichenbaum 1992a; Suzuki et al. 1993; Goulet and Murray 2001). An alternative, Parallel Model (Fig. 1B), holds that recognition memory for modalities other than vision relies on separate sensory-specific processes outside the perirhinal region (Kowalska et al. 2001; Wan et al. 2001; Fritz et al. 2005; Winters and Reid 2010). This second model would, however, need to accommodate evidence that the perirhinal region can integrate object information from different senses (Goulet and Murray 2001; Holdstock et al. 2009; Winters and Reid 2010). Determining the correct model will appreciably advance our understanding of the basic organization of recognition memory.

Rats with perirhinal cortex lesions were tested for object recognition using a task that combines elements of delayednonmatching-to-sample (multiple trials, continuous testing) with the preferential exploration of novel objects (Albasser et al. $2010 a, b)$. Rats are allowed to explore successive pairs of objects, one novel and one familiar, at alternating ends of a maze (Albasser et al. 2010a,b). As all objects (both novel and familiar) are in fixed locations and cover baited food wells, to ensure the objects are initially contacted by the rats, the protocol also

\footnotetext{
${ }^{3}$ Corresponding author.
}

E-mail AlbasserM@cf.ac.uk.

Article is online at http://www.learnmem.org/cgi/doi/10.1101//m.2132911. facilitates the testing of recognition memory in the dark as rats will readily locate the test objects (Albasser et al. 2010a). Recognition memory was first examined for pairs of complex "junk" objects that provide multisensory (visual, somatosensory, olfactory) cues (Experiment 1). Next, the recognition of novel from familiar odors was assessed (Experiment 2). Recognition memory for objects composed of the same material (Duplo) but having different appearances followed (Experiment 3). These Duplo objects have similar olfactory and tactile surface properties, but different visual features (in the light) and unique threedimensional shapes. In all three experiments, testing in the light and in the dark was counterbalanced.

Throughout Experiments 1-3 the novel object on a given trial became the familiar object for the next trial (as it has just been explored). This running recognition design makes it possible to examine the transfer of multimodal sensory information across consecutive trials, e.g., will an object made familiar in the light be recognized when experienced on the next trial in the dark? Experiment 4, therefore, examined whether rats with perirhinal cortex lesions can switch between available cue classes when solving such object recognition problems (e.g., light to dark, dark to light). Although the Convergent Model predicts lesion-induced deficits across all recognition conditions in Experiments 1-4, the Parallel Model predicts selective recognition deficits.

\section{Results}

The overall findings were both clear and consistent. Rats with perirhinal cortex lesions (PRh) were consistently impaired when tested in the light both for the recognition of complex objects (Experiment 1) and for the recognition of objects distinguished by a combination of their visual and shape cues (Experiment 3, Duplo objects). When the same rats were tested for object recognition in the dark their performance significantly improved such that they often did not differ from the Control rats. The PRh 


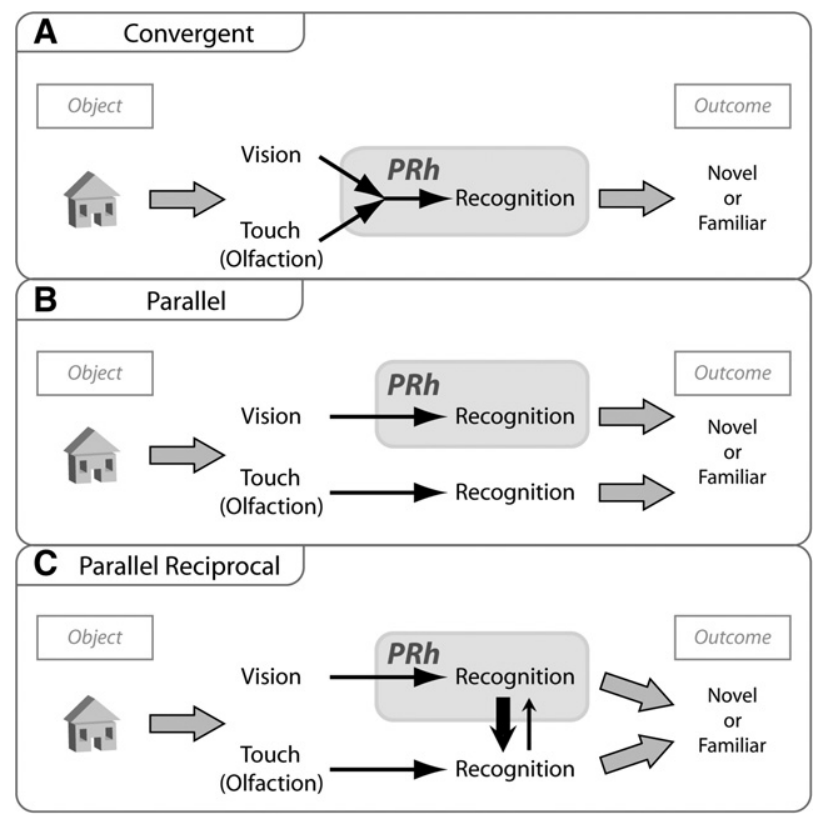

Figure 1. Schematic diagram showing hypothetical models of the interactions between sensory recognition systems. (A) Convergent: Perirhinal cortex receives information for recognition from different senses. (B) Parallel: Different sensory recognition systems work in parallel with the perirhinal cortex involved only in vision. For Model B it is assumed that the detection of novelty by any one stream is sufficient to make a "new" decision. (C) Parallel Reciprocal: The perirhinal cortex is vital for the visual system but also interacts with other sensory systems. For example, vision might override touch or olfaction (bold arrow between the two systems). Olfaction is assumed to mirror the organization for touch, and so is placed in parentheses. The converging arrows represent multisensory systems that are then unitized prior to making the decision whether the object is novel or familiar.

rats also showed intact odor recognition when tested in the dark (Experiment 2), though neither the PRh nor the control rats showed effective odor recognition in the light.

Recognition scores were derived by measuring the preferential exploration of the novel of two stimuli. The principal analyses refer to the "D2" index of recognition (Ennaceur and Delacour 1988). D2 is the difference in time spent exploring novel and familiar objects divided by total exploration time. Additional analyses examined the total amounts of exploration (in seconds). Experiments 1-3 involved counterbalanced sessions, one in the light and one in the dark (10 trials per session, retention delay between sample and test of up to $60 \mathrm{sec}$ ), whereas in Experiment 4 all four conditions occurred within the same session in a counterbalanced sequence (Table 1; see Materials and Methods).

\section{Experiment 1: Object recognition memory (light/dark)}

The recognition of junk objects was examined in two different sessions (10 trials each), one in the light and one in the dark (Table 1A). Perirhinal cortex lesions were far more disruptive for object recognition in the light than in the dark (Fig. 2). Although the PRh group was significantly impaired in the dark (simple effects; $F_{(1,40)}=7.30, P<0.001$ ) as well as in the light $\left(F_{(1,40)}=64.2, P<0.001\right)$, there was a significant lesion by condition interaction $\left(F_{(1,20)}=13.48, P=0.002\right)$. This interaction reflected the improved performance by the PRh group when tested in the dark (simple effects; $F_{(1,20)}=22.22, P<0.001$ ), which contrasted with the Controls who performed at comparable levels in the light and the dark $(F<1)$. One sample $t$-tests showed that the PRh group discriminated the novel from the familiar objects in the dark $\left(t_{(11)}=11.78, P<0.001\right)$. Further, despite their deficits, the PRh group also discriminated above chance in the light $\left(t_{(11)}=8.69, P<0.001\right)$. For total times spent exploring the objects (novel plus familiar) there was no group difference $\left(F_{(1,20)}=3.20, P=0.09\right)$, and no difference between light and dark $\left(F_{(1,20)}=2.77, P=0.11\right)$. Inspection of the trial-by-trial data (Fig. 2) showed how the use of multiple trials can both reveal and strengthen any group differences, whereas analyses based on D2 scores for individual trials failed to find any evidence that the profile of performance across the sessions differed between the two groups (group-by-trial interaction, $F<1$ ).

\section{Experiment 2: Odor recognition memory (light/dark)}

Different odors were presented to the animals for recognition in either the light or the dark in two counterbalanced sessions (10 trials each). The odors were released from plastic cubes that were visually indistinguishable (Table $1 \mathrm{~A}$ ). In contrast to Experiment 1 , the PRh rats did not differ overall from their controls $(F<1)$. Although neither group preferred the novel odors in the light (Fig. 3) (one sample $t$-tests, both $t<1$ ), both groups showed improved performance in the dark $\left(F_{(1,20)}=4.87, P=0.04\right)$ and there was no lesion by condition interaction $(F<1)$. Both groups now discriminated the novel odor in the dark (one sample $t$-test; Control rats, $t_{(9)}=4.02, P=0.003 ;$ PRh rats, $t_{(11)}=5.57, P<$ $0.001)$. For total exploration times there was no overall lesion effect and no difference between light and dark (both $F<1$ ). The trial-by-trial data (Fig. 3) suggest that experience of the testing regime in the dark aided odor recognition in the PRh group. This possibility was examined using the individual trial data (i.e., not

Table 1. Presentation arrangement of the objects for the different recognition protocols

(A) Experiments 1-3: Recognition memory (object, odor, shape)

$\begin{array}{llllllllllll}\text { Trials } & 0 & 1 & 2 & 3 & 4 & 5 & 6 & 7 & 8 & 9 \\ \text { Objects } & - & \text { A } & \text { B } & \text { C } & \text { D } & \text { E } & \text { F } & \text { G } & \text { H } & \text { I } & \text { J } \\ & \text { A } & \text { B } & \text { C } & \text { D } & \text { E } & \text { F } & \text { G } & \text { H } & \text { I } & \text { J } & \text { K }\end{array}$

(B) Experiment 4: Object recognition memory-transfer between light and dark

\begin{tabular}{llllllllllllllllllllll} 
Trials & 0 & 1 & 2 & 3 & 4 & 5 & 6 & 7 & 8 & 9 & 10 & 11 & 12 & 13 & 14 & 15 & 16 & 17 & 18 & 19 & 20 \\
Objects & - & $\mathrm{A}$ & $\mathrm{B}$ & $\mathrm{C}$ & $\mathrm{D}$ & $\mathrm{E}$ & $\mathrm{F}$ & $\mathrm{G}$ & $\mathrm{H}$ & $\mathrm{I}$ & $\mathrm{J}$ & $\mathrm{K}$ & $\mathrm{L}$ & $\mathrm{M}$ & $\mathrm{N}$ & $\mathrm{O}$ & $\mathrm{P}$ & $\mathrm{Q}$ & $\mathrm{R}$ & $\mathrm{S}$ & $\mathrm{T}$ \\
& $\mathbf{A}$ & $\mathbf{B}$ & $\mathbf{C}$ & $\mathbf{D}$ & $\mathbf{E}$ & $\mathbf{F}$ & $\mathbf{G}$ & $\mathbf{H}$ & $\mathbf{I}$ & $\mathbf{J}$ & $\mathbf{K}$ & $\mathbf{L}$ & $\mathbf{M}$ & $\mathbf{N}$ & $\mathbf{O}$ & $\mathbf{P}$ & $\mathbf{Q}$ & $\mathbf{R}$ & $\mathbf{S}$ & $\mathbf{T}$ & $\mathbf{U}$ \\
\hline
\end{tabular}

Schematic showing the object sequences used in Experiments 1-4. On every trial the rat is exposed to two objects: one novel (in bold) and the other familiar, as it occurred in the previous trial. (A) In Experiments 1-3 all rats had one session in the light and one session in the dark (counterbalanced). (B) In Experiment 4 the lighting was repeatedly changed within the session so that two consecutive trials were in the light, two in the dark, and so on. Trials in the dark are shown

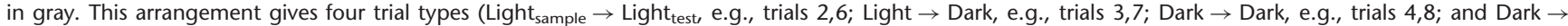
Light, e.g., trials 5,9). All sessions began with familiarization of the initial object (trial 0 ). 


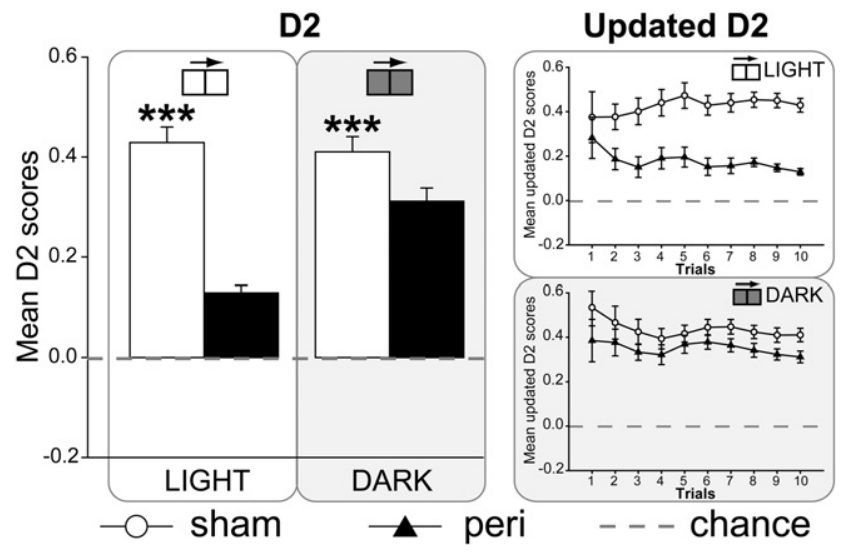

Figure 2. Experiment 1: Object recognition of junk objects in the light and dark by rats with perirhinal cortex lesions (black) and surgical controls (white). Rats with perirhinal lesions are particularly impaired when tested in the light. The histograms (left) show the D2 scores when the data from all 10 trials are considered; the graphs (right) show the updated D2 scores over successive trials. D2 is the time exploring the novel object minus the time exploring the familiar object, divided by total exploration. Scores can range from +1 to -1 . Data shown are mean \pm standard error of the mean. Group differences ${ }^{* * *} p<0.001$.

the cumulative data), but no significant evidence was found of a group-by-trial interaction $\left(F_{(9,180)}=1.14, P=0.34\right)$.

\section{Experiment 3: Visual and shape recognition memory (light/dark)}

Object recognition was tested in the light and in the dark across two different sessions (10 trials each) (Table 1A), but now all objects were assembled from the same material (Duplo, LEGO). Consequently, all objects shared textural and odor properties but differed in their visual appearance and three-dimensional structure.

The perirhinal lesions severely impaired performance in the light (Fig. 4) so that the PRh rats failed to prefer the novel object (one sample $t$-test; $\mathrm{D} 2: t_{(11)}=1.58, P=0.14$ ). In contrast, the perirhinal lesions had no apparent effect on recognition performance in the dark, where both groups successfully recognized the novel objects (one sample $t$-test, both groups $P<0.001$ ). As a consequence, there was a lesion by condition interaction $\left(F_{(1,20)}=\right.$ $5.98, P=0.02)$ reflecting the PRh group deficit in the light (simple effects; $\left.F_{(1,40)}=19.95, P<0.001\right)$ but not in the dark $(F<1)$. Once again, there was no lesion effect on total exploration times $(F<1)$ although overall, animals spent more time exploring objects in the light than in the dark $\left(F_{(1,20)}=6.72, P=0.017\right)$. Again, inspection of the trial-by-trial data showed how the use of multiple trials helped to strengthen those group differences.

\section{Experiment 4: Object recognition memory-transfer between light and dark}

The test consisted of a single session of 20 trials that used junk objects throughout. Two consecutive trials in the light were followed by two consecutive trials in the dark, and so on (see Table 1B). Thus, four different pairs of trial types were analyzed: (1) sample in light, test in light $(\mathrm{L} \rightarrow \mathrm{L})$, (2) sample in light, test in dark $(\mathrm{L} \rightarrow \mathrm{D})$, (3) sample in dark, test in dark $(\mathrm{D} \rightarrow \mathrm{D})$, and (4) sample in dark, test in light $(\mathrm{D} \rightarrow \mathrm{L}$ ) (see Materials and Methods for more detail). The control rats performed at a similar level for all four conditions (simple effects: $F_{(3,60)}<1$ ) (Fig. 5).
Overall, the PRh rats were very impaired $\left(F_{(1,20)}=80.6, P<\right.$ 0.001 ), but inspection of the results (Fig. 5) shows that their performance on the Dark $\rightarrow$ Dark condition was qualitatively different to the other three conditions: In the Dark $\rightarrow$ Dark condition the PRh group recognized the novel objects (one sample $t$-test; $\left.t_{(11)}=6.05, P<0.001\right)$, but failed to do so in any of the other three conditions (one sample $t$-test; $\mathrm{L} \rightarrow \mathrm{L}: t_{(11)}<1 ; \mathrm{L} \rightarrow \mathrm{D}: t_{(11)}=1.33$, $\left.P=0.21 ; \mathrm{D} \rightarrow \mathrm{L}: t_{(11)}=1.95, P=0.078\right)$. For Dark $\rightarrow$ Dark, the PRh and Control rats had similar scores, whereas the PRh rats were severely impaired on the other three conditions. This pattern explains the lesion by test condition interaction $\left(F_{(3,60)}=3.26\right.$, $P=0.03)$. Simple effects confirmed that although the Control group had higher D2 scores for all three conditions involving the light (each condition $P<0.001$ ), the two groups did not differ for Dark $\rightarrow$ Dark $\left(F_{(3,80)}=1.05, P=0.3\right)$. Further, the two groups neither differed on overall exploration times $\left(F_{(1,20)}=1.19, P=\right.$ $0.2)$ nor was there any evidence of a group difference for any of the four separate conditions $\left(F_{(3,60)}=1.20, P=0.31\right)$.

\section{Histology}

The boundaries of the perirhinal cortex were taken from Burwell (2001). Four of the original 16 PRh rats were excluded from all analyses as three had excessive cortical damage and one had only unilateral perirhinal cortex damage. The percentage of cell loss in the perirhinal cortex and the neighboring areas in the remaining 12 rats is presented in Table 2 . In these 12 rats the surgeries removed almost the entire perirhinal cortex (Fig. 6), although there was often limited sparing at its most rostral border $(n=10)$. The PRh lesions often extended ventrally to involve adjacent parts of the caudal piriform and the rostral lateral entorhinal cortices, sometimes in both hemispheres. The extent of extrahippocampal damage is presented in Table 2 . Immediately dorsal to the perirhinal cortex is ventral area $\mathrm{Te} 2$, which was sometimes thinned $(n=7)$. In eight rats the lesions extended medially to cause a very restricted patch of cell loss in caudal CA1, immediately adjacent to the fundus of the rhinal sulcus (bilateral in two cases). The lesions occasionally extended unilaterally onto more superior cortex $(n=4)$. In five cases there was unilateral damage
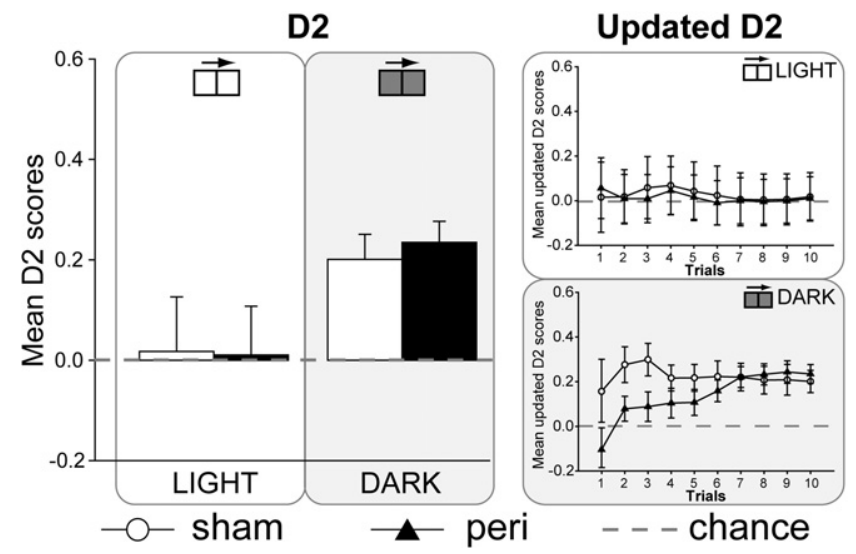

Figure 3. Experiment 2: Odor recognition in the light and dark by rats with perirhinal cortex lesions (black) and surgical controls (white). Rats with perirhinal lesions were not impaired, though neither group showed novel odor recognition in the light. The histograms (left) show the D2 scores when the data from all 10 trials are considered; the graphs (right) show the updated D2 scores over successive trials. D2 is the time exploring the novel object minus the time exploring the familiar object, divided by total exploration. Scores can range from +1 to -1 . Data shown are mean \pm standard error of the mean. 


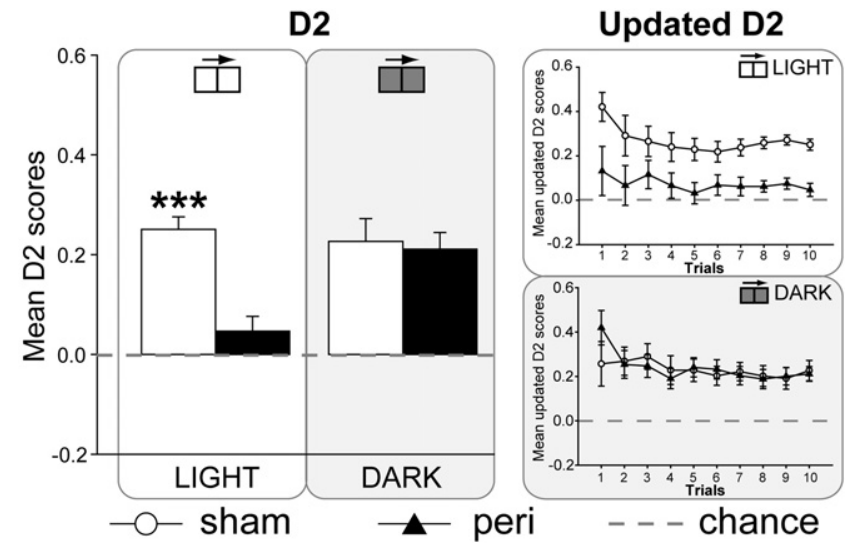

Figure 4. Experiment 3: Visual and shape recognition (Duplo objects) in the light and in the dark by rats with perirhinal cortex lesions (black) and surgical controls (white). Rats with perirhinal lesions were only impaired when tested in the light. The histograms (left) show the D2 scores when the data from all 10 trials are considered; the graphs (right) show the updated D2 scores over successive trials. D2 is the time exploring the novel object minus the time exploring the familiar object, divided by total exploration. Scores can range from +1 to -1 . Data shown are mean \pm standard error of the mean. Group differences ${ }^{* * *} p<0.001$.

to the rostral post-rhinal cortex. Subsequent analyses were carried out in order to test whether the overall extent of perirhinal damage influenced the animals' performance (D2) in Experiment 1 (Object recognition memory in the light) and in Experiment 3 (Object recognition in the light). No significant correlation was found between lesion size and D2 (Experiment 1, $r=-0.13, P=$ 0.68 ; Experiment $3 r=-0.35, P=0.26$ ).

\section{Discussion}

Four complementary experiments analyzed how different classes of sensory information interact and compete to support recognition memory. In all experiments, recognition memory was measured indirectly by examining the preference for novel over familiar stimuli. The behavior of the control rats is considered first.

Experiments 1 and 4 showed that normal rats are equally proficient at recognizing "junk" objects in the light and in the dark, the latter condition revealing their effective use of tactile and, presumably, olfactory cues. The ability of the same rats to use just olfactory cues (Experiment 2) and just tactile shape information (Duplo recognition, Experiment 3) was then confirmed with recognition memory tests in the dark. An unexpected result was the failure of the control rats in Experiment 2 to show olfactory recognition memory when tested in the light. This failure cannot be due to the particular odors used or their order of presentation as these were counterbalanced across the dark and light conditions. Instead, this recognition failure (i.e., discriminating novel odor cubes in the dark but not in the light) points to competition from salient visual cues, such as the plastic odor cubes themselves, which were all identical in appearance and so could block differential exploration.

Other experiments have shown that normal rats are capable of solving olfactory recognition problems in the light (Fortin et al. 2002), and so it is interesting to consider why visual cues impaired performance in the present study. One possible reason is that all training in the light (Experiments 1 and 2) reinforced the use of vision to guide the rats to the objects and, thereby, to the underlying rewards. This pattern of prior training, combined with the fact that the rats were always allowed to freely explore the odor cubes may have led to visual properties gaining control. This study is, in fact, not the first example of illumination levels critically affecting the use of olfactory cues. Experiments run in a radial-maze in either the light or the dark, and with either the presence or absence of additional olfactory cues, clearly showed how olfactory cues were only used to guide accurate arm choice when the rats were in the dark (Lavenex and Schenk 1995). Likewise, previous studies have shown how visuospatial cues can outweigh olfactory cues when the two are in conflict (Olton and Collison 1979; Suzuki et al. 1980). The present study showed a similar phenomenon but for recognition rather than spatial learning.

The flexibility of recognition memory is highlighted in Experiment 4, where control rats switched from Light $\rightarrow$ Dark and from Dark $\rightarrow$ Light between sample and test. As the same tactile cues were present in the light and in the dark, these switches do not provide a pure test of cross-modal matching (Goulet and Murray 2001; Winters and Reid 2010), which is the ability to match object identities by transferring information across sensory modalities. Consequently, the present experimental conditions are different from those in a recent study that examined tactile object recognition (in the dark), visual object recognition (objects were behind a transparent screen so removing tactile cues), and then used a Dark (tactile) $\rightarrow$ Light (vision) switch to demonstrate cross-modal matching (Winters and Reid 2010). In contrast, the present testing arrangement leaves multiple sensory cues in the light conditions, so allowing cue competition. This competition is important as it captures the more naturalistic situation of repeatedly encountering the same multisensory object but under different conditions, and facilitates comparisons of models based on sensory integration or competition. Despite these differences, both studies found that normal rats could solve such problems while perirhinal lesions impair Dark $\rightarrow$ Light object transformations (Winters and Reid 2010). The present testing arrangements also made it possible, for the first time, to compare directly Light $\rightarrow$ Dark with Dark $\rightarrow$ Light recognition switches for rats.

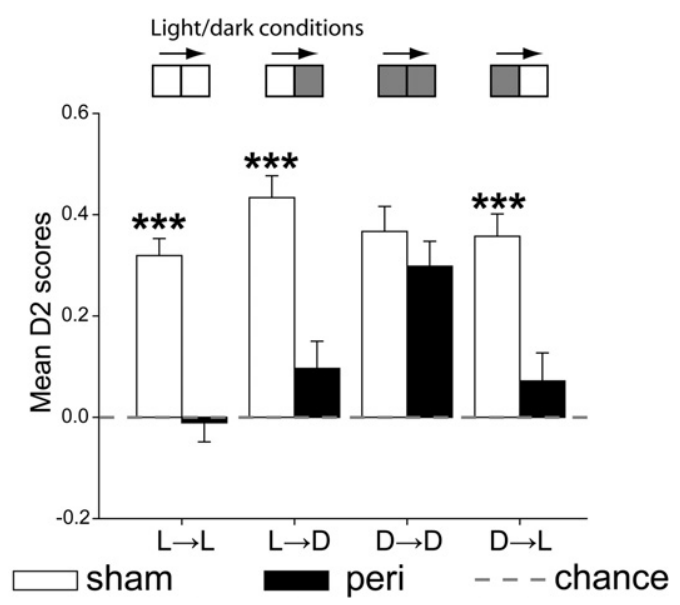

Figure 5. Experiment 4: Object recognition-transfer between object sampling in the light or in the dark to a recognition test in the light or the dark. The resulting four conditions were: light to light $(L \rightarrow L)$, light to dark $(L \rightarrow D)$, dark to dark $(D \rightarrow D)$, and dark to light $(D \rightarrow L)$. Rats with perirhinal lesions (black histograms) were consistently impaired whenever sampled or tested in the light, but performed at normal levels for $D \rightarrow D$. The histograms show the D2 scores when the data from all five trials at each condition are combined. D2 is the time exploring the novel object minus the time exploring the familiar object, divided by total exploration. Scores can range from +1 to -1 . Data shown are mean \pm standard error of the mean. Group differences $* * * P<0.001$. 
Table 2. Extent of damage in the perirhinal cortex and the neighboring areas

\begin{tabular}{|c|c|c|c|c|}
\hline $\begin{array}{l}\text { Percentage of } \\
\text { damage }\end{array}$ & $100 \%-75 \%$ & $75 \%-50 \%$ & $50 \%-25 \%$ & $25 \%-0 \%$ \\
\hline $\begin{array}{l}\text { Rostral perirhinal } \\
\text { cortex }\end{array}$ & 0 & 2 & 7 & 3 \\
\hline $\begin{array}{l}\text { Mid perirhinal } \\
\text { cortex }\end{array}$ & 8 & 4 & 0 & 0 \\
\hline $\begin{array}{l}\text { Caudal perirhinal } \\
\text { cortex }\end{array}$ & 10 & 2 & 0 & 0 \\
\hline $\begin{array}{c}\text { Area TEv_at } \\
\text { lesion AP }\end{array}$ & 0 & 0 & 6 & 6 \\
\hline Area TEv-total & 0 & 0 & 0 & 12 \\
\hline $\begin{array}{l}\text { Piriform cortex- } \\
\text { at lesion AP }\end{array}$ & 0 & 0 & 2 & 10 \\
\hline $\begin{array}{l}\text { Piriform cortex- } \\
\text { total }\end{array}$ & 0 & 0 & 0 & 12 \\
\hline $\begin{array}{l}\text { Lateral entorhinal } \\
\text { cortex-at } \\
\text { lesion AP }\end{array}$ & 3 & 5 & 4 & 0 \\
\hline $\begin{array}{l}\text { Lateral entorhinal } \\
\text { cortex-total }\end{array}$ & 0 & 0 & 0 & 12 \\
\hline
\end{tabular}

The distribution of damage in the 12 rats with bilateral lesions of the perirhinal cortex (Burwell 2001). The percentage of damage was allocated to one of four categories, each of $25 \%$. For each area, the number represents the number of rats falling into one of the four categories. The bold numbers correspond to the modal number for each brain region. The $100 \%-75 \%$ category reflects extensive damage, whereas the $25 \%-0 \%$ category reflects considerable sparing. The perirhinal cortex was divided into its rostral, mid, and caudal parts. The percentage of extra-perirhinal damage was measured in three adjacent regions using both the size of that region adjacent to the perirhinal cortex (antero-posterior from bregma $=-2.76,-6.84$ ) and its overall (total) area (see Materials and Methods).

Despite switching between different illuminations from sample to test (Light $\rightarrow$ Dark, Dark $\rightarrow$ Light), control rats performed at a level that was comparable to when both the sample and the test were in the light or in the dark. The ability of normal rats to switch from Light $\rightarrow$ Dark is possibly the less remarkable if it is assumed that vision does not compete with the simultaneous acquisition of nonvisual (tactile) information as these sources of information complement one another to create a representation of the object, e.g., via cross-modal matching. (In this way the situation is very different from the odor recognition task, where the odors do not inform about the visual appearance of the container.) This tactile information then supports novelty discrimination when switched to the dark. More challenging is the Dark $\rightarrow$ Light condition where at choice rats are confronted with two objects in the light, only one of which has been previously experienced, and that only in the dark. As neither object has been viewed in the light before, at choice both the familiar and the novel object provide novel visual features. Given their high D2 scores (Fig. 5) it would seem that normal rats are able to ignore any novel visual cues and either rely on nonvisual information to guide their recognition memory performance, i.e., nonvisual systems retain control (Fig. 1A) as signals of visual novelty are canceled out between the two test stimuli, or rats rely on cross-modal matching (Over and Mackintosh 1969; Winters and Reid 2010) so that previous tactile and present visual cues are integrated and unitized when moving into the light.

Rats with perirhinal lesions behaved very abnormally on these various tests of recognition. Consistent with numerous previous studies, spontaneous object recognition memory in the light was severely impaired (Aggleton et al. 1997; Ennaceur and Aggleton 1997; Norman and Eacott 2004; Winters and Bussey
2005; Winters et al. 2008), reflecting the failure of visual recognition memory mechanisms. There was not, however, a complete failure of recognition memory mechanisms, as shown by the spared novelty discrimination of the PRh rats when all testing was in the dark (Experiments 1-4) (for a similar result, see also Winters and Reid 2010). This contrast in performance between light and dark was most clear-cut in the last two experiments. In Experiment 3 (Duplo objects), the PRh and Control groups showed equivalent recognition memory performance when tested in the dark, where both groups presumably discriminated the novel stimuli based on tactile (whisking) information. The PRh rats did not, however, show novelty discrimination in the light, even though these rats should have been able to use tactile cues again to solve the problem. Experiment 4 then showed that perirhinal lesions disrupt performance irrespective of whether objects are initially sampled in the light and then tested in the dark (Light $\rightarrow$ Dark) or sampled in the dark and then tested in the light (Dark $\rightarrow$ Light). As this task does not demand crossmodal matching, it seems remarkable that the PRh rats performed at chance for all but the Dark $\rightarrow$ Dark condition, i.e., the rats could potentially have relied on tactile/olfactory cues throughout. Their failure to do so indicates not only the interplay between recognition memory systems, but also reveals that the loss of the perirhinal cortex leads to a strong bias toward the visual system for both encoding and retrieval, i.e., even when effective tactile cues are available. This visual bias in the PRh rats is not apparent in the control rats as they can readily perform the Dark $\rightarrow$ Light condition, where a bias toward visual cues would result in reduced discrimination. The visual bias in the PRh rats is only released in the absence of visual information at either encoding or retrieval. This failure of the PRh rats to integrate cue types in a flexible

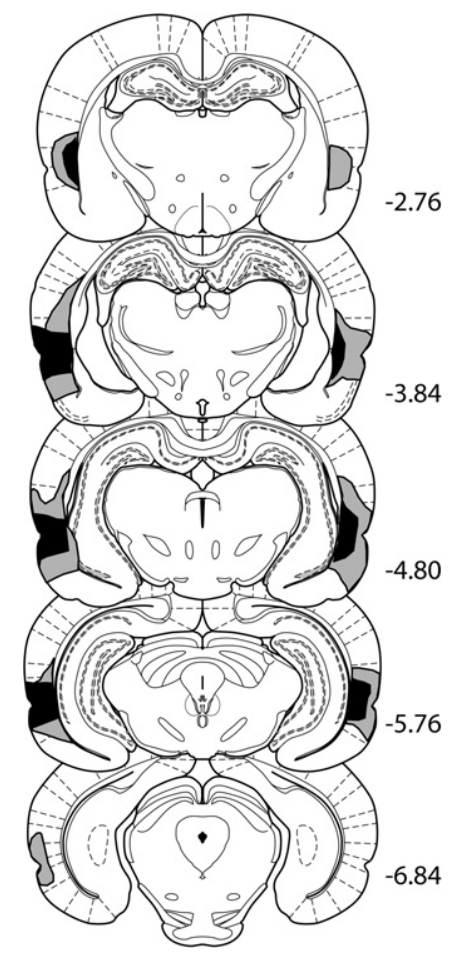

Figure 6. Diagrammatic reconstructions of the perirhinal cortex lesions showing the individual cases with the largest (gray) and smallest (black) lesions. The numbers refer to the distance (in millimeters) from bregma (adapted, with permission of Elsevier $(1)$ 2005, from Paxinos and Watson 2005). The PRh group comprised 12 rats. 
manner may also help to explain contextual fear conditioning deficits that are observed after perirhinal lesions when the contexts to be discriminated contain multisensory information with overlapping cues (Bucci et al. 2002). The implication is that sensory integration within the rat perirhinal cortex normally helps to distinguish complex objects (Furtak et al. 2007; Winters and Reid 2010) and that vision has a key role in this unitization process.

The spontaneous object recognition task (Ennaceur and Delacour 1988), which measures the preferential exploration of novel objects when set alongside familiar objects, is now a mainstay of batteries for the cognitive assessment of rodents (Winters et al. 2008). The standard task uses complex objects that could allow rats to use tactile and olfactory cues, in addition to visual cues. For this reason, the current study reveals new insights into the interplay between sensory systems supporting performance of this multisensory task. As an example, the present experiments help to explain the perplexing problem of why rats with perirhinal cortex lesions show intact object recognition memory in the dark (Winters and Reid 2010), yet are exceptionally poor at object recognition memory in the light (Ennaceur and Aggleton 1997; Norman and Eacott 2004; Winters and Bussey 2005; Winters et al. 2008) when in the latter condition they should still be able to rely on tactile information. Other implications are that even if manipulations designed to disrupt perirhinal cortex function, e.g., cortical drug infusions (Warburton et al. 2003; Winters and Reid 2010) only have their effects on visual processes, this should be sufficient to control recognition memory across different senses in the light.

The precise importance of the perirhinal cortex for olfactory recognition memory requires further examination. A number of additional sites, including the piriform cortex, entorhinal cortex, orbital frontal cortex, hippocampus, and medial dorsal thalamic nucleus, are potentially key loci for this function, and all are directly connected with the perirhinal cortex (Burwell and Amaral 1998; Burwell and Witter 2002). It has for example, been proposed that parahippocampal areas (entorhinal and perirhinal) can maintain longer-term representations of odors that then support recognition memory (Otto and Eichenbaum 1992a,b). Evidence for this suggestion includes the finding that combined perirhinal-entorhinal lesions impair olfactory delayed-nonmatching-to-sample over longer retention intervals (Otto and Eichenbaum 1992a), whereas removal of just the lateral entorhinal cortex spares olfactory recognition memory (Wirth et al. 1998). Meanwhile, electrophysiological studies have found that perirhinal cells are activated during odor sampling, many of which show odor-selective activity across a delay period (Young et al. 1997). Even so, the present study found no direct evidence that the perirhinal cortex is necessary for olfactory recognition, although the use of longer retention intervals would be required to test fully the model of Otto and Eichenbaum (1992a). The implication is that a number of sites may together support this function, with the caveat that the detection of a novel odor may be different from using odor in combination with other sensory modalities (e.g., vision, tactile) to help determine the novelty or familiarity of a complex object, which is the focus of the present study. In the latter situation, the rat perirhinal cortex may still prove to have an important role (e.g., Experiment 4).

Of the two models of object recognition memory outlined in the Introduction, the present results contradict a Convergent Model with multiple sensory recognition mechanisms within the perirhinal cortex (Fig. 1A). This convergent account fails to predict the normal levels of performance in the PRh rats when sample and test are in the dark, i.e., there should be a tactile/olfactory recognition deficit. In contrast, the results support the notion of separate, parallel mechanisms outside the perirhinal cortex to support tactile and olfactory recognition memory (see also Winters and Reid 2010). To this list can be added audition, as other animal studies indicate that auditory recognition memory does not depend on the perirhinal cortex (Kowalska et al. 2001; Wan et al. 2001; Fritz et al. 2005). The Parallel Model (Fig. 1B) does, however, require refinement to permit competition between modalities as revealed, for example, by the odor recognition experiment (Fig. 1C). The Parallel Model also breaks down for rats with perirhinal lesions as the PRh rats in Experiment 4 should be able to solve the Dark $\rightarrow$ Light problem: The PRh rats can acquire object tactile information (as demonstrated by the Dark $\rightarrow$ Dark test) yet are then unable to use that information effectively in the light. This failure occurs because of the paradoxical ability of vision to control choice behavior in the absence of the perirhinal cortex, even though in this manipulation (Dark $\rightarrow$ Light) vision will not distinguish novel from familiar objects. The result is the need to move to a more dynamic model ("Parallel Reciprocal Model") (Fig. 1C) that permits some forms of integration and competition across modalities, yet still permits the various modalities to work separately.

\section{Materials and Methods}

\section{Animals}

The four recognition experiments used 26 male, Lister Hooded rats (Harlan, Bicester, UK), housed in pairs under diurnal conditions (14 h light/10 h dark). Water was provided ad libitum throughout the study. Prior to surgery, rats weighed between 282 and $322 \mathrm{~g}$. After a 2-wk recovery period, animals were fooddeprived up to $85 \%$ of their free-feeding body weight and behavioral testing began. All experiments were performed in accordance with the UK Animals (Scientific Procedures) Act, 1986 and associated guidelines. As a consequence, the experiments adhered to the U.S. Public Health Service's Policy on Humane Care and Use of Laboratory Animals (PHS policy) and the Guide for the Care and Use of Laboratory Animals (the Guide).

\section{Surgery}

Sixteen rats received bilateral perirhinal cortex lesions (PRh) and 10 rats served as controls. Rats were anesthetized using an isoflurane-oxygen mixture before placement in a stereotaxic frame (David Kopf Instruments), with the incisor bar set at $5.0 \mathrm{~mm}$ to the horizontal plane. A sagittal incision was made in the scalp, and the skin retracted to expose the skull. The perirhinal cortex lesions were made by injecting a solution of $0.09 \mathrm{M}$ $\mathrm{N}$-methyl-D-aspartic acid (NMDA; Sigma) dissolved in phosphatebuffered saline (pH 7.4) in three sites per hemisphere using a 1- $\mu \mathrm{L}$ Hamilton syringe held with a microinjector (Kopf Instruments, Model 5000). Bilateral injections of $0.225 \mu \mathrm{L}$ NMDA were made at a rate of $0.10 \mu \mathrm{L} / \mathrm{min}$ with the needle left in place for a further $4 \mathrm{~min}$. The coordinates of the three injections relative to bregma were as follows: (1) antero-posterior (AP) -1.8 , medial-lateral (ML) \pm 5.9 , dorso-ventral (DV) -9.3; (2) AP - 3.4, ML \pm 6.1 , DV -9.6, and (3) AP -5.0, ML \pm 6.2 , DV -9.0. Rats in the surgical control group received identical treatment with the exception that the dura was repeatedly perforated with a 25-gauge Microlance 3 needle (Becton Dickinson), but no fluid was infused into the brain.

\section{Histology}

Following behavioral testing, all rats received a lethal overdose of sodium pentobarbitone $(60 \mathrm{mg} / \mathrm{kg}$, Euthatal) and then transcardially perfused with $0.1-\mathrm{M}$ phosphate buffer saline (PBS) followed by $4 \%$ paraformaldehyde in $0.1 \mathrm{M}$ PBS (PFA). The brains were removed and post-fixed in PFA for $4 \mathrm{~h}$ and then transferred to $25 \%$ sucrose overnight at room temperature with rotation. Sections were cut at $40 \mu \mathrm{m}$ on a freezing microtome in the coronal 
plane and then stained with cresyl violet. Cytoarchitectural borders for the perirhinal cortex were taken from Burwell (2001).

\section{Volumetric analyses}

Estimates were made of the extent of the lesions in all $12 \mathrm{PRh}$ animals. Coronal sections were viewed on a Leica DMRB microscope, photographed using an Olympus DP70 camera and the images transferred to a computer. Lesion measurements were carried out using the program analySIS ${ }^{\wedge} \mathrm{D}$ (Soft-Imaging Systems). A set of three bilateral, standard coronal sections (rostral, mid, and caudal) were first constructed according to the cytoarchitectonic divisions of Burwell (2001). Lesion borders were then drawn in a frame area including the perirhinal cortex (areas 35 and 36), area TEv, and the piriform cortex or the entorhinal cortex (depending on AP level). The perirhinal cortex was subdivided into three subregions: rostral (posterior to AP -2.76 in relation to bregma) (Paxinos and Watson 2005), mid (posterior to AP -3.84), and caudal (posterior to AP -4.80). It should be noted that our rostral perirhinal measurements would include much of the posterior insular cortex as described by Shi and Cassell (1999), who proposed a much more restricted perirhinal region than Burwell (2001).

For each of the three subregions of the perirhinal cortex (rostral, mid, caudal), measurements were taken from four consecutive sections from each hemisphere immediately caudal to each of these AP levels. Consequently, the extent of the perirhinal lesion was mapped out on 12 coronal sections along the anterior-posterior extent of the perirhinal cortex. The tissue loss from all 24 hemispheres was summed to produce a total lesion size and was expressed as a percentage compared to the size of an intact perirhinal cortex (control rat). For the neighboring areas, the extent of the damage was (1) measured at lesion AP (between -2.76 and -5.76 from bregma) and (2) measured compared to the entire size of the regions, e.g., the borders of the piriform cortex go from $\mathrm{AP}+4.20$ to $\mathrm{AP}-3.84$ (Paxinos and Watson 2005).

\section{Apparatus}

Rats were tested in a bow-tie shaped maze made with steel walls and a wooden floor (see Fig. 7A). The maze was $120 \mathrm{~cm}$ long, $50 \mathrm{~cm}$ wide, and $50 \mathrm{~cm}$ high. Each end of the apparatus was triangular, the apices of which were joined by a narrow corridor $(12 \mathrm{~cm}$ wide). An opaque sliding door set in the middle of the corridor could be raised by the experimenter. The far wall of each triangle

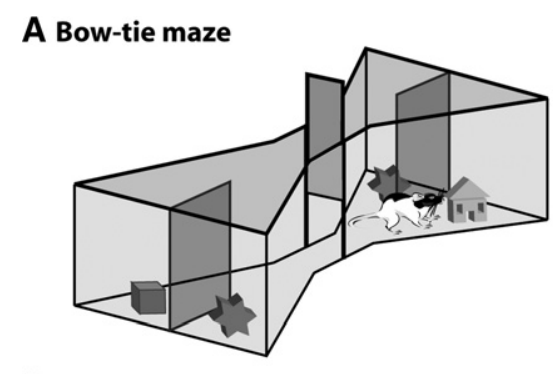

\section{B Objects}

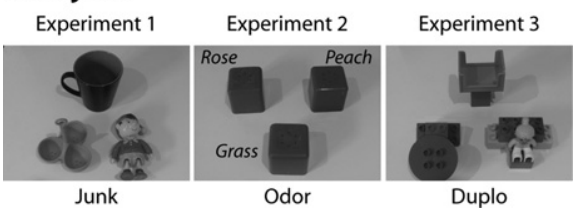

Figure 7. (A) Schematic of the bow-tie maze. A central sliding door separates the two ends of the maze in which two objects are placed. (B) Photographs showing examples of objects used in Experiment 1 -junk objects (left); Experiment 2-odor cubes (middle; visually identical but with different aromas, e.g., rose, peach, or grass); Experiment 3-Duplo objects (right). contained two recessed food wells, $3.5 \mathrm{~cm}$ in diameter and $2 \mathrm{~cm}$ deep. The food wells were separated by a short, opaque dividing wall that protruded $15 \mathrm{~cm}$ from the middle of the end wall. These food wells were covered by objects in the experiment proper.

For all light sessions, illumination was provided by ceiling lights giving a mean light intensity of 581.0 lX in the center of the maze. For the dark sessions, all sources of light were switched off or blocked, resulting in a light intensity of $0.11 \mathrm{lX}$ in the center of the maze. The experimenter wore night vision goggles (Productive Firm Dipol Ltd.) and the session was recorded with two infrared cameras (Maplin Electronics) fixed directly above the maze.

\section{Objects}

Experiments 1 and 4 used 43 different pairs of junk objects, each differing in shape, texture, size, and color (Fig. 7B). Any object with an obvious smell was excluded. In Experiment 2, blue plastic cubes containing different aromas (e.g., peach, rose) were used (Vortex Cubes, Dale Air). All 22 pairs of cubes $(5 \mathrm{~cm} \times 5 \mathrm{~cm} \times$ $5 \mathrm{~cm}$ ) looked identical. The top of each cube was pierced with numerous holes to dispense the aroma (Fig. 7B). In Experiment 3 , objects were solely made of Duplo (bricks and special elements, LEGO). Thus, all 22 pairs of Duplo objects shared similar textures and odors, but varied in their three-dimensional shapes and visual appearance. For all experiments, every object was large enough to cover a food well and light enough to be displaced by a rat. Every object had a duplicate that was used on the following trial. All objects (Experiments 1-4) were cleaned with alcohol wipes after each session.

\section{Behavioral testing-general protocol}

Following pretraining (see Albasser et al. 2010a), all rats received multiple trials during which the animal could freely explore two objects, one novel and one familiar (Fig. 7A). To start each session (Table 1A), the rat was placed on one side of the maze with no objects and no food reward. The central sliding door was then raised and the rat was allowed to run to the other side of the maze where a single object (object A) covered a food well that contained a single sucrose pellet ( $45 \mathrm{mg}$, Noyes Purified Rodent Diet). The rat remained in that part of the maze (with object A) for $1 \mathrm{~min}$ (Table 1A). The sliding door was then raised and the rat ran to the opposite side of the maze. There, the rat had a free choice between object A, now familiar, and novel object B (Trial 1, Table 1A). Both objects A and B covered single sucrose pellets, and were concurrently available for the rat to explore for $1 \mathrm{~min}$. The central door was then raised (Trial 2) to reveal object B (now familiar) and object C (novel). This procedure continued, e.g., Trial 3 involved object C (now familiar) vs. object D (novel), up to Trial 10 (Experiments 1-3) or Trial 20 (Experiment 4).

Baiting of both the novel and familiar objects encouraged the animals' object explorations, but did not affect test validity as this relied on differential exploration and not on learning a rule as in a delayed-nonmatching-to-sample task. The placement of the novel object varied from left to right according to a pseudorandom schedule, whereas the order of the particular objects used in the tests was reversed for half of the rats. This counterbalancing meant that the "novel" object in any given object pair was reversed for half the rats.

\section{Behavioral testing-Experiment 1: Object recognition (light/dark)}

All rats received two sessions: one in the light and one in the dark (Table 1A). The testing protocol was identical to that described previously (General Protocol). Two different sets of 11 pairs of junk objects were used in both sessions to provide 10 trials per session (Fig. 7B). Half of the animals were run first in the light, whereas the other half started in the dark. 


\section{Behavioral testing-Experiment 2: Odor recognition (light/dark) and Experiment 3: Visual and shape recognition (light/dark)}

The protocols were identical to Experiment 1 (Table 1A), except that instead of junk objects, odor cubes (Experiment 2) and Duplo objects (Experiment 3) were used (Fig. 7B). As before, the light/dark conditions were counterbalanced.

\section{Behavioral testing-Experiment 4: Transfer between light and dark}

All rats received a single session of 20 trials (Table 1B). Half of the animals started Trials 1 and 2 in the light, with Trials 3 and 4 in the dark and Trials 5 and 6 back in the light, etc. The remaining rats started the first two trials in the dark in order to counterbalance the order of the light/dark conditions. There were four different conditions, each given for five trials (Table 1B): (1) from light to light $(\mathrm{L} \rightarrow \mathrm{L})$, (2) from light to dark ( $\rightarrow \mathrm{D})$, (3) from dark to dark $(\mathrm{D} \rightarrow \mathrm{D})$, and (4) from dark to light $(\mathrm{D} \rightarrow \mathrm{L})$. The moment when the light was switched on or off was carefully chosen to ensure that rats could not cheat. Thus, when the rats went from light to dark $(\mathrm{L} \rightarrow \mathrm{D})$ the room illumination was removed just before raising the central door. When the rats went from dark to light $(\mathrm{D} \rightarrow \mathrm{L})$ the illumination was increased just after closing the central door, i.e., after the rat had entered the "light" end of the apparatus.

\section{Analysis of behavior}

Animals were video-recorded throughout testing. Analysis of behavior was always scored by an investigator who was blind to which rats were in the Control and the PRh groups. Object exploration was defined as directing the nose at a distance $<1 \mathrm{~cm}$ from the object, with the vibrissae moving, and/or touching it with the nose or the paws. Object exploration was not scored when rats sat on the object or when rats used the object to rear upward. Two discrimination indices (D1 and D2) were calculated (Ennaceur and Delacour 1988). D1 is the difference in exploration time devoted to the novel object vs. the familiar object, and was cumulatively summed across trials. D2 uses the difference in exploration times (i.e., D1) but then divides D1 by the total duration of exploration given to both novel and familiar objects. The resulting ratio can vary between +1 and -1 , with positive D2 scores showing a preference for novel objects. The D2 score after every trial was recalculated using the cumulative data ("updated D2"). The updated D2 on the last trial is equivalent to the mean D2 of the entire test. Because the standard object recognition test in the bow-tie maze allows the testing of animals across multiple trials within a session, the updated D2 scores revealed how the rats' cumulative performances progressed along the session.

\section{Statistical analysis}

Analyses are presented for D2, but parallel analyses were conducted for D1, which gave the same array of significant and nonsignificant results. For each experiment, the discrimination scores (D2) for the PRh and Control groups were analyzed using a one between- (lesion; perirhinal or control) by a one within- (condition; light or dark) ANOVA. When significant interactions were found the simple effects were analyzed as recommended by Winer (1971) using the pooled error term. When there was a significant main effect but no interaction, simple effects were examined so that conditions (light, dark) that significantly differed between groups could be identified (Howell 1987). One sample $t$-tests were conducted using the discrimination scores (D1 and D2) to determine if either group performed above chance (zero), i.e., showed a preference for novelty. These one sample $t$-tests were one-tailed as the only issue was whether scores were above chance. The significance level was set throughout at $P<0.05$.

Trial-by-trial data are presented for Experiments 1-3 (Figs. $2-4$ ) as these results show how the use of cumulative data from multiple trials helps to reduce variance within each group and reveal any systematic shifts in performance within a session. Statistical analyses are not typically presented for these trialby-trial data as the "Updated D2" is derived from cumulative scores, i.e., the D2 scores at different trials are not independent.

\section{Acknowledgments}

This work was supported by the Wellcome Trust (WT087855).

\section{References}

Aggleton JP, Keen S, Warburton EC, Bussey TJ. 1997. Extensive cytotoxic lesions of the rhinal cortices impair recognition but spare spatial alternation in the rat. Brain Res Bull 43: 279-287.

Albasser MM, Chapman RJ, Amin E, Iordanova MD, Vann SD, Aggleton JP. 2010a. New behavioral protocols used to extend our knowledge of rodent object recognition memory. Learn Mem 17: 407-419.

Albasser MM, Poirier GL, Aggleton JP. 2010b. Qualitatively different modes of perirhinal-hippocampal engagement when rats explore novel vs. familiar objects as revealed by c-fos imaging. Eur J Neurosci 31: $134-147$.

Brown MW, Aggleton JP. 2001. Recognition memory: What are the roles of the perirhinal cortex and hippocampus? Nat Rev Neurosci 2: 51-61.

Bucci DJ, Saddoris MP, Burwell RD. 2002. Contextual fear discrimination is impaired by damage to the postrhinal or perirhinal cortex. Behav Neurosci 116: 479-488.

Burwell RD. 2001. Borders and cytoarchitecture of the perirhinal and postrhinal cortices in the rat. J Comp Neurol 436: 17-41.

Burwell RD, Amaral DG. 1998. Cortical afferents of the perirhinal, postrhinal, and entorhinal cortices of the rat. J Comp Neurol 398: 179-205.

Burwell RD, Witter MP. 2002. Basic anatomy of the parahippocampal region in monkeys and rats. In The parahippocampal region (ed. MP Witter, FG Wouterlood), pp. 35-59. Oxford University Press, New York.

Ennaceur A, Aggleton JP. 1997. The effects of neurotoxic lesions of the perirhinal cortex combined to fornix transection on object recognition memory in the rat. Behav Brain Res 88: 181-193.

Ennaceur A, Delacour J. 1988. A new one-trial test for neurobiological studies of memory in rats. 1: Behavioral data. Behav Brain Res 31: 47-59.

Fortin NJ, Agster KL, Eichenbaum HB. 2002. Critical role for the hippocampus in memory for sequences of events. Nat Neurosci 5: 458-462.

Fritz J, Mishkin M, Saunders RC. 2005. In search of an auditory engram. Proc Natl Acad Sci 102: 9359-9364.

Furtak SC, Wei S-M, Agster KL, Burwell RD. 2007. Functional neuroanatomy of the parahippocampal region in the rat: The perirhinal and postrhinal cortices. Hippocampus 17: 709-722.

Goulet S, Murray EA. 2001. Neural substrates of crossmodal association memory in monkeys: The amygdala versus the anterior rhinal cortex. Behav Neurosci 115: 271-284.

Holdstock JS, Hocking J, Notley P, Devlin JT, Price CJ. 2009. Integrating visual and tactile information in the perirhinal cortex. Cereb Cortex 19: 2993-3000.

Howell DC. 1987. Statistical methods for psychology. Duxberry Press, Belmont, CA.

Kowalska DM, Kusmierek P, Kosmal A, Mishkin M. 2001. Neither perirhinal/entorhinal nor hippocampal lesions impair short-term auditory recognition memory in dogs. Neuroscience 104: 965-978.

Lavenex P, Schenk F. 1995. Integration of olfactory information in a spatial representation enabling accurate arm choice in the radial arm maze. Learn Mem 2: 299-319.

Mumby DG, Pinel JP. 1994. Rhinal cortex lesions and object recognition in rats. Behav Neurosci 108: 11-18.

Murray EA, Mishkin M. 1986. Visual recognition in monkeys following rhinal cortical ablations combined with either amygdalectomy or hippocampectomy. J Neurosci 6: 1991-2003.

Norman G, Eacott MJ. 2004. Impaired object recognition with increasing levels of feature ambiguity in rats with perirhinal cortex lesions. Behav Brain Res 148: 79-91.

Olton DS, Collison C. 1979. Intramaze cues and 'odor trails' fail to direct choice behavior on an elevated maze. Anim Learn Mem 7: 221-223.

Otto T, Eichenbaum H. 1992a. Complementary roles of the orbital prefrontal cortex and the perirhinal-entorhinal cortices in an odor-guided delayed-nonmatching-to-sample task. Behav Neurosci 106: $762-775$.

Otto T, Eichenbaum H. 1992b. Neuronal activity in the hippocampus during delayed non-match to sample performance in rats: Evidence for hippocampal processing in recognition memory. Hippocampus 2: 323-334. 
Over R, Mackintosh NJ. 1969. Cross-modal transfer of intensity discrimination by rats. Nature 224: $918-919$.

Paxinos G, Watson C. 2005. The rat brain in stereotaxic coordinates, 5 th ed. Academic, San Diego.

Shi CJ, Cassell MD. 1999. Perirhinal cortex projections to the amygdaloid complex and hippocampal formation of the rat. J Comp Neurol $\mathbf{4 0 6}$ 299-328.

Suzuki S, Augerinos G, Black AH. 1980. Stimulus control of spatial behaviour on the eight-arm maze in rats. Learn Motiv 11: 1-18.

Suzuki WA, Zola-Morgan S, Squire LR, Amaral DG. 1993. Lesions of the perirhinal and parahippocampal cortices in the monkey produce long-lasting memory impairment in the visual and tactile modalities. J Neurosci 13: 2430-2451.

Wan H, Warburton EC, Kusmiereck P, Aggleton JP, Kowalska DM, Brown MW. 2001. Fos imaging reveals differential neuronal activation of areas of rat temporal cortex by novel and familiar sounds. Eur $J$ Neurosci 14: $118-124$.

Warburton EC, Koder T, Cho K, Massey PV, Duguid G, Barker GRI, Aggleton JP, Bashir ZI, Brown MW. 2003. Cholinergic neurotransmission is essential for perirhinal cortical plasticity and recognition memory. Neuron 38: 987-996.

Winer MP. 1971. Statistical principles in experimental design. McGraw-Hill, New York.

Winters BD, Bussey TJ. 2005. Transient inactivation of perirhinal cortex disrupts encoding, retrieval, and consolidation of object recognition memory. J Neurosci 25: 52-61.

Winters BD, Reid JM. 2010. A distributed cortical representation underlies crossmodal object recognition in rats. J Neurosci 30: 6253-6261.

Winters BD, Saksida LM, Bussey TJ. 2008. Object recognition memory: Neurobiological mechanisms of encoding, consolidation and retrieval. Neurosci Biobehav Rev 32: 1055-1070.

Wirth S, Ferry B, Di Scala G. 1998. Facilitation of olfactory recognition by lateral entorhinal cortex lesion in rats. Behav Brain Res 91: 49-59.

Young BJ, Otto T, Fox GD, Eichenbaum H. 1997. Memory representation within the parahippocampal region. J Neurosci 17: 5183-5195.

Received December 23, 2010; accepted in revised form March 13, 2011. 


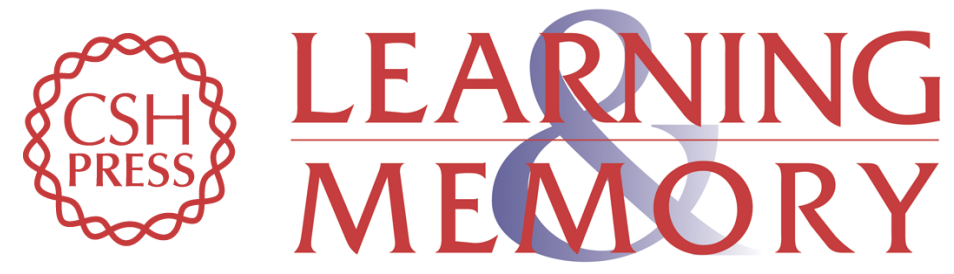

\section{Separate but interacting recognition memory systems for different senses: The role of the rat perirhinal cortex}

Mathieu M. Albasser, Eman Amin, Mihaela D. Iordanova, et al.

Learn. Mem. 2011, 18:

Access the most recent version at doi:10.1101//m.2132911

References This article cites 33 articles, 7 of which can be accessed free at:

http://learnmem.cshlp.org/content/18/7/435.full.html\#ref-list-1

License

Email Alerting Receive free email alerts when new articles cite this article - sign up in the box at the Service top right corner of the article or click here. 